\title{
Tricuspid and Mitral Valve Regurgitation with Bi-fascicular Block Following a Horse Kick
}

\author{
Tomoki Kokubun ${ }^{1}$, Masayoshi Oikawa ${ }^{1}$, Yasuhiro Ichijo ${ }^{1}$, Yoshiyuki Matsumoto ${ }^{1}$, \\ Tetsuro Yokokawa ${ }^{1}$, Kazuhiko Nakazato ${ }^{1}$, Yoshiyuki Sato ${ }^{2}$, Shinya Takase ${ }^{2}$, Hiroharu Shinjo ${ }^{2}$, \\ Hitoshi Yokoyama ${ }^{2}$, Hitoshi Suzuki ${ }^{1}$, Shu-ichi Saitoh ${ }^{1}$ and Yasuchika Takeishi ${ }^{1}$
}

\begin{abstract}
:
A 40-year-old man was transferred to our hospital following an isolated horse kick injury to the anterior chest wall. The case showed bi-fascicular block, severe tricuspid valve regurgitation due to ruptured chordae tendineae of the anterior leaflet, moderate mitral valve regurgitation due to prolapse of mitral anterior leaflet, and hypokinetic motion of the inferior septal wall. Both tricuspid and mitral insufficiency were completely repaired by a surgical operation. Fortunately, these injuries were not fatal in this case, but the comprehensive assessment of cardiac damage and careful observation are important for managing patients with cardiac injury.
\end{abstract}

Key words: blunt chest trauma, myocardial contusion, tricuspid valve regurgitation, mitral valve regurgitation, bi-fascicular block

(Intern Med 57: 1597-1600, 2018)

(DOI: 10.2169/internalmedicine.9762-17)

\section{Introduction}

Non-penetrating cardiac trauma causes many cardiac complications, including myocardial contusion, chordae and papillary muscle rupture, coronary artery dissection or thrombosis, various arrhythmias, septal rupture, and free wall rupture $(1,2)$.

Motor vehicle accidents are the most common cause, but other accidents, including falls from heights, sports accidents, bomb injuries, and animal kicks, can cause cardiac trauma (3).

The tricuspid valve is the most commonly affected valve because of the anterior location of the right ventricle $(4,5)$, but a few cases of multiple valve injuries with conduction disturbance have been reported.

We herein report a case of tricuspid and mitral valve regurgitation with bi-fascicular block following a horse kick.

\section{Case Report}

A previously healthy 40-year-old man was transferred to our hospital following an isolated horse kick injury to his anterior chest wall during the Soma Nomaoi wild horse chase festival (Shinto ritual in the Soma District of Fukushima Prefecture). His consciousness level was normal, and his vital signs were as follows: blood pressure 78/43 $\mathrm{mmHg}$, heart rate 61 beats per minute, and oxygen saturation $96 \%$ on room air.

Focused assessment with sonography for trauma showed no intraperitoneal effusion, but slight pericardial effusion. Auscultation of the heart revealed holosystolic murmur of Levine II/VI at 4th intercostal space of left sternal border. Electrocardiography (ECG) showed a regular sinus rhythm with complete right bundle branch block (CRBBB) and left anterior fascicular block. He had had undergone a health examination one month before the accident, and his ECG findings were normal at that time (Fig. 1).

\footnotetext{
${ }^{1}$ Department of Cardiovascular Medicine, Fukushima Medical University, Japan and ${ }^{2}$ Department of Cardiovascular Surgery, Fukushima Medical University, Japan

Received: July 3, 2017; Accepted: August 29, 2017; Advance Publication by J-STAGE: January 11, 2018

Correspondence to Dr. Tomoki Kokubun, kokutomo@fmu.ac.jp
} 
(a)

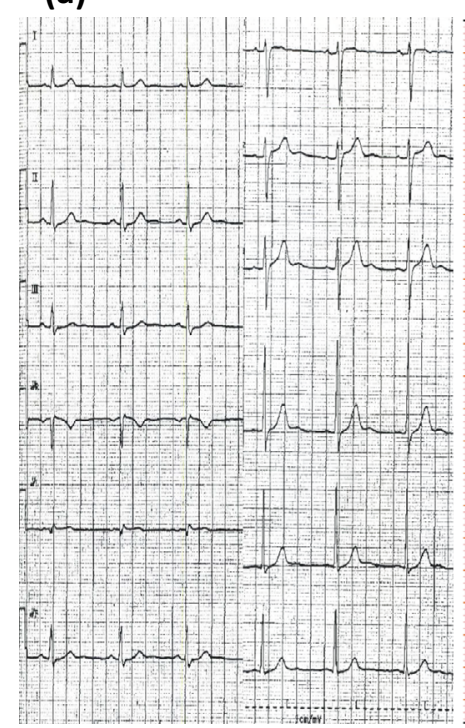

(b)

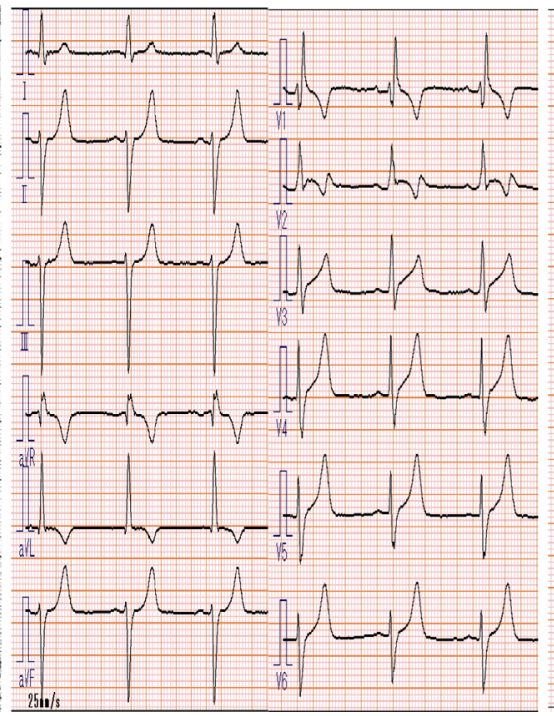

(c)

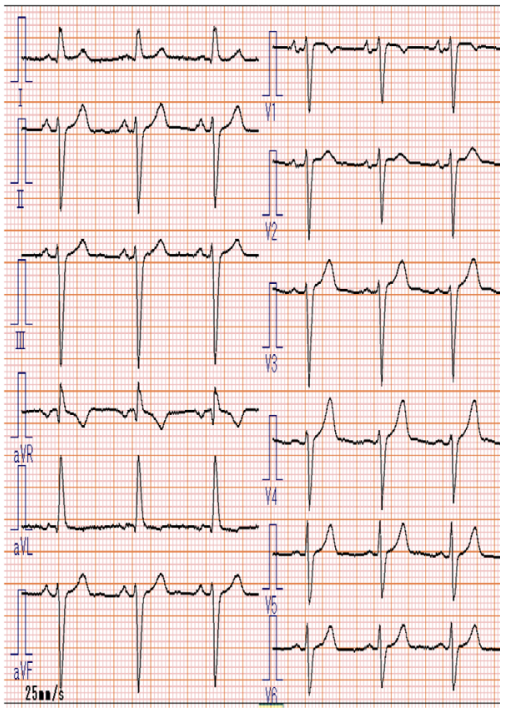

Figure 1. Changes in ECG findings. (a) ECG at one month before the accident. (b) ECG on admission to our hospital. (c) ECG on day 4 after the accident.

(a)

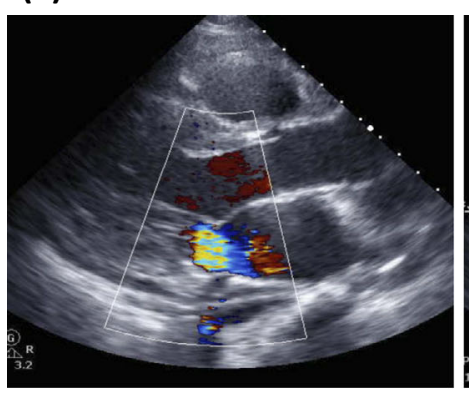

(d) (b)

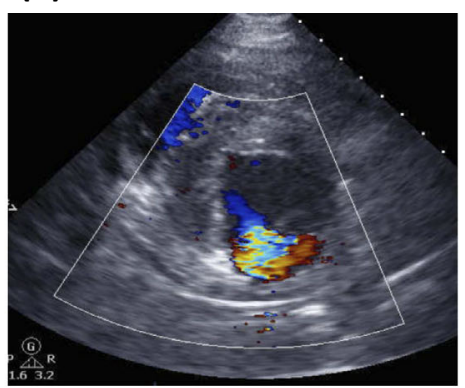

(e)
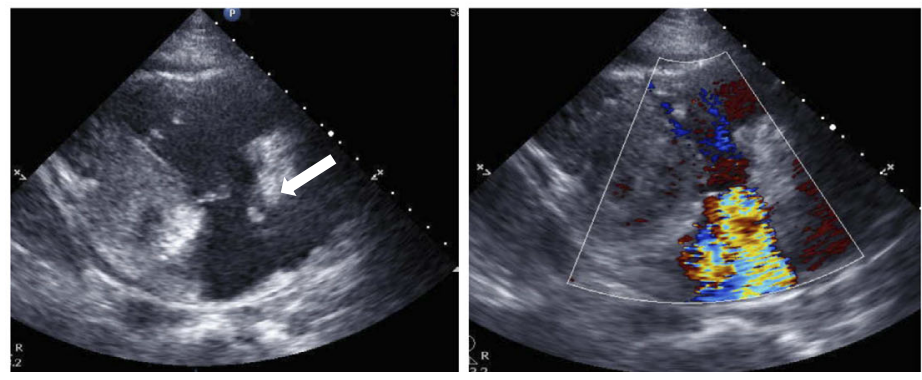

Figure 2. Echocardiographic findings on admission. (a, b) Moderate mitral valve regurgitation (MR). (c) Transesophageal echocardiography (TEE) revealed prolapse of the medial side of the mitral anterior leaflet (arrow) with moderate MR jet. (d, e) Parasternal long-axis view of right ventricular inflow. Severe tricuspid valve regurgitation due to ruptured chordae tendineae of the anterior leaflet (arrow) was detected.

Echocardiography showed enlargement of the right side heart and severe tricuspid valve regurgitation (TR) due to ruptured chordae tendineae of the anterior leaflet. Moderate mitral valve regurgitation (MR) with prolapse of the medial side of the anterior mitral leaflet (AML) was also recognized. In addition, the inferior septal wall near the posteromedial papillary muscle showed severe hypokinetic motion (Fig. 2).

Computed tomography (CT) revealed sternal fracture at the level of the right ventricular outflow tract, and contrast defect in the inferior septal wall of the left ventricle (Fig. 3). Coronary computed tomography angiography revealed neither coronary artery atherosclerosis, coronary dissection nor hematoma, indicating that myocardial perfusion was impaired due to myocardial contusion. His blood pressure recovered to $108 / 62 \mathrm{mmHg}$ after drip infusion of extracellular fluid. The serum cardiac enzyme levels increased to $943 \mathrm{mg} /$ $\mathrm{dL}$ for creatine kinase and $106 \mathrm{ng} / \mathrm{mL}$ for creatine kinase- 
(a)

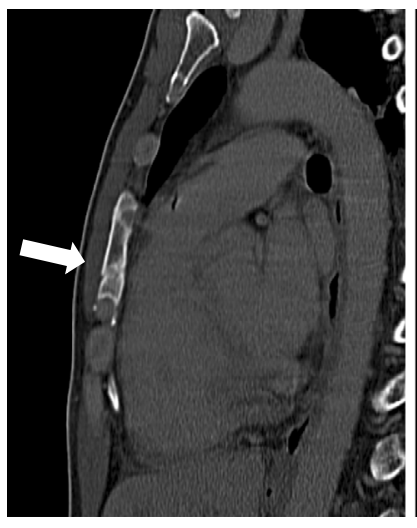

(b)

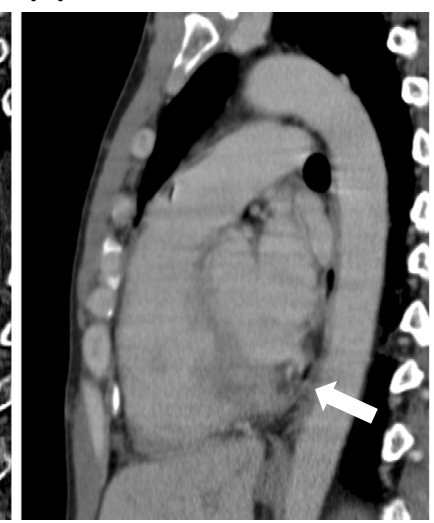

(c)

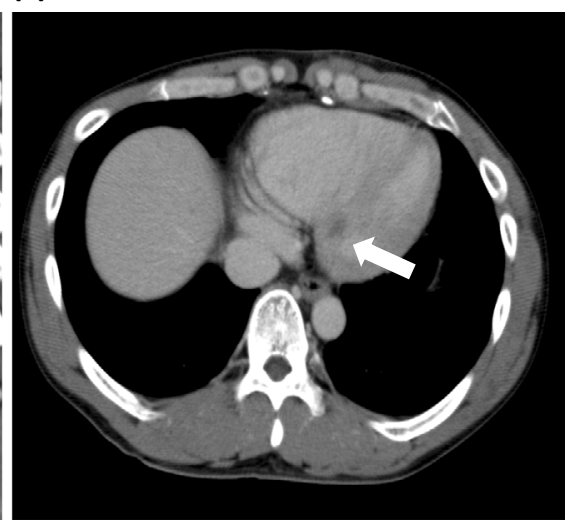

Figure 3. The findings of computed tomography (CT) on admission. (a) Plane CT showed sternal fracture at the level of the right ventricular outflow tract (arrow). (b, c) Enhanced CT demonstrated the defect of contrast medium in the inferior septal wall of the left ventricle (arrows).

(a)

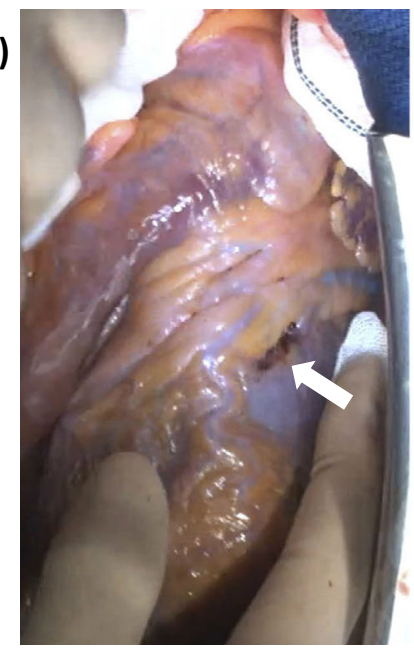

(b)

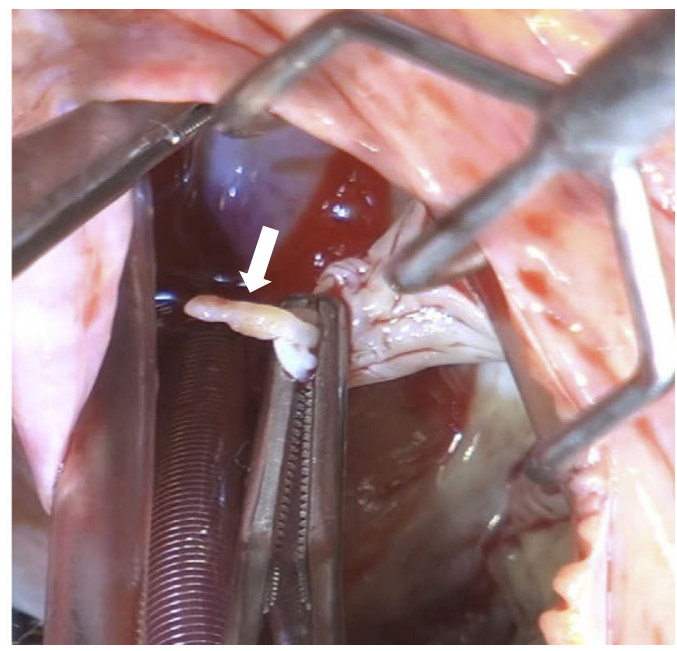

Figure 4. Operative findings. (a) A $20 \times 10-\mathrm{mm}$ epicardial defect at the left side of the left anterior descending artery (arrow). (b) Prolapse of the anterior leaflet of tricuspid valve with a ruptured papillary muscle (arrow).

myocardial band (MB) fraction on the second day of illness. Although the patient presented with slight heart failure on the third day, it was treated with a small amount of diuretic drug. CRBBB disappeared spontaneously on the fourth day of illness, but left anterior fascicular block remained.

Initially, there were no signs of worsening mitral and tricuspid valve regurgitation, and the patient did not desire to receive surgery. However, right heart dilatation progressed gradually, and this case was eventually indicated for surgery because of symptomatic primary severe TR due to blunt chest trauma and progressive right ventricular dysfunction. In addition, this case showed moderate MR due to AML medial prolapse. Therefore, we performed tricuspid valvuloplasty and mitral valve ring-annuloplasty 46 days after the trauma. Intraoperative findings revealed hemorrhagic pericardial effusion and a $20 \times 10-\mathrm{mm}$ epicardial defect at the left side of the left anterior descending artery. Consistent with the echocardiographic findings, the medial side of the anterior mitral leaflet and anterior leaflet of the tricuspid valve were prolapsed (Fig. 4). We initially suspected partial tearing of the papillary muscle supporting the anterior mitral leaflet, but it was unclear on operating findings. There was no evidence of elongation or degeneration of the mitral leaflets. We suspected that the myocardial contusion had caused the lack of contraction of the papillary muscle, leading to the partial prolapse of AML. Because mitral valve annuloplasty alone improved coaptation of mitral leaflets, and MR disappeared, we did not perform artificial chordae implantation.

Post-operative echocardiography showed no regurgitation at the mitral or tricuspid valves, and the patient was discharged on the 14th post-operative day.

\section{Discussion}

An impact of a horse's kick delivers more than 10,000 N to the body, causing fractures of the skull and other bones as well as devastating damage to the intestines (6). Several 
reports have shown that horses' kicks induce cardiac injury, including cardiac rupture, tricuspid insufficiency, and complete AV block (7-9). The mechanisms underlying cardiac injury due to blunt chest trauma are as follows: rapid direct compression between sternum and vertebra increases the intrathoracic pressure, and excessive pressure at the phase of isochoric contraction injured atrioventricular valve, chordae tendineae, and papillary muscle (10). In the present case, traumatic lesions namely, at the tricuspid valve, medial side of the mitral valve, and inferior septal wall were located between the line of the sternum and vertebra, supporting this mechanism.

ECG abnormalities caused by blunt chest trauma correlate with cardiac complications (11). Right bundle branch block is observed most frequently because the tricuspid valve and right heart muscle are directly damaged by blunt chest trauma (12). Arrhythmias and conduction defects are usually transient with the recovery of cardiac damage (13), but some cases require permanent implantation of a pacemaker device (14).

In the present case, the first assessment of echocardiography and ECG revealed not only apparent tricuspid regurgitation but relatively small mitral regurgitation, pericardial effusion, and bi-fascicular block. Fortunately, none of the injuries were fatal in this case, but such injuries should be watched carefully because several complications, including valve insufficiency, cardiac tamponade, and complete atrioventricular block, can occur in the subacute phase of trauma $(15,16)$. In this case, the pre-operative findings were as follows: 1) severe TR due to ruptured chordae tendineae of the anterior leaflet, 2) moderate MR with AML prolapse of the medial side, 3) sternal fracture at the level of the right ventricle (RV) outflow tract, 4) myocardial contusion in the left ventricle (LV) inferior septal, and 5) RV and RA- dilatation that progressed gradually. The surgical indications were symptomatic primary severe TR due to blunt chest wall trauma and progressive RV dysfunction. In addition, the case showed moderate MR due to AML medial prolapse. The comprehensive assessment of cardiac damage and careful observation are important for managing patients with cardiac injury.

\section{Conclusion}

We experienced a case of tricuspid and mitral valve regurgitation with bi-fascicular block following a horse kick. The careful assessment and observation are important for manag- ing patients with cardiac injury.

The authors state that they have no Conflict of Interest (COI).

\section{References}

1. Prêtre R, Chilcott M. Blunt trauma to the heart and great vessels. N Engl J Med 336: 626-632, 1997.

2. Sybrandy KC, Cramer MJ, Burgersdijk C. Diagnosing cardiac contusion: old wisdom and new insights. Heart 89: 485-489, 2003.

3. Bock JS, Benitez RM. Blunt cardiac injury. Cardiol Clin 30: 545555, 2012.

4. Parmley LF, Manion WC, Mattingly TW. Nonpenetrating traumatic injury of the heart. Circulation 18: 371-396, 1958.

5. Liedtke AJ, DeMuth WE. Nonpenetrating cardiac injuries. Am Heart J 86: 687-697, 1973.

6. Exadaktylos AK, Eggli S, Inden P, Zimmermann H. Hoof kick injuries in unmounted equestrians. Improving accident analysis and prevention by introducing an accident and emergency based relational database. Emerg Med J 19: 573-575, 2002.

7. Gorman R, Nuttall SM. Traumatic right ventricular rupture following a horse kick. BMJ Case Rep 2012: bcr2012006657, 2012.

8. Knobloch K, Rossner D, Strüber M, Fischer S, Leyh RG, Haverich A. Traumatic tricuspid insufficiency after horse kick. J Trauma 56: 694-696, 2004.

9. Benitez RM, Gold MR. Immediate and persistent complete heart block following a horse kick. Pacing Clin Electrophysiol 22: 816818, 1999.

10. Miyoshi Y, Ohara K. Traumatic cardiac injury. Kyobu Geka 57: 742-750, 2004 (in Japanese, Abstract in English).

11. Maenza RL, Seaberg D, D'Amico F. A meta-analysis of blunt cardiac trauma: ending myocardial confusion. Am J Emerg Med 14: 237-241, 1996.

12. Hachiro Y, Sugimoto S, Takagi N, Osawa H, Morishita K, Abe T. Native valve salvage for post-traumatic tricuspid regurgitation. J Heart Valve Dis 10: 276-278, 2001 (in Japanese, Abstract in English).

13. Pontillo D, Capezzuto A, Achilli A, Serraino L, Savelli S, Guerra R. Bifascicular block complicating blunt cardiac injury. A case report and review of the literature. Angiology 45: 883-890, 1994.

14. Aykan AC, Oguz AE, Yildiz M, Özkan M. Complete atrioventricular block associate with non-penetrating cardiac trauma in a 40year-old man. J Emerg Med 44: e41-e43, 2013.

15. Farmery AD, Chambers PH, Banning AP. Delayed rupture of the mitral valve complicating blunt chest trauma. J Accid Emerg Med 15: 422-423, 1998.

16. Liang HM, Chen QL, Zhang EY, Hu J. Sternal fractures and delayed cardiac tamponade due to a severe blunt chest trauma. Am J Emerg Med 34: 758.e1-758.e3, 2016.

The Internal Medicine is an Open Access article distributed under the Creative Commons Attribution-NonCommercial-NoDerivatives 4.0 International License. To view the details of this license, please visit (https://creativecommons.org/licenses/ by-nc-nd/4.0/).

\section{(C) 2018 The Japanese Society of Internal Medicine} Intern Med 57: 1597-1600, 2018 